(C) [2009] IEEE. Reprinted, with permission, from [Steven W. Su, Brian D.O. Anderson, Weidong Chen, and Hung T. Nguyen, Multi-realization of nonlinear systems, Decision and Control, 2009 held jointly with the 2009 28th Chinese Control Conference. CDC/CCC 2009. Proceedings of the 48th IEEE Conference on 15-18 Dec. 2009]. This material is posted here with permission of the IEEE. Such ermission of the IEEE does not in any way imply IEEE endorsement of any of the University of Technology, Sydney's products or services. Internal or personal use of this material is permitted. However, permission to reprint/republish this material for advertising or promotional purposes or for creating new collective works for resale or redistribution must be obtained from the IEEE by writing to pubspermissions@ieee.org. By choosing to view this document, you agree to all provisions of the copyright laws protecting it 


\title{
Multi-realization of nonlinear systems
}

\author{
Steven W. Su, Brian D.O. Anderson, Weidong Chen, and Hung T. Nguyen
}

\begin{abstract}
The system multi-realization problem is to find a state-variable realization for a set of systems, sharing as many parameters as possible. A multi-realization can be used to efficiently implement a multi-controller architecture for multiple model adaptive control. We extend the linear multi-realization problem to nonlinear systems. The problem of minimal multirealization of a set of MIMO systems is introduced and solved for feedback linearizable systems.
\end{abstract}

\section{INTRODUCTION}

For the implementation of multiple model adaptive control (MMAC) [1] [2] [6] [14] using switching between a finite number of distinct controllers [12] [13], Morse [12] [13] proposed (for the SISO case) a new concept, viz. the multi-controller can be efficiently implemented by using a parameter-dependent feedback structure around a single fixed set of components of the controller. As argued in [12], because at any instant of time only one of the finite set of possible controllers is to be applied to the plant, it is only necessary to generate one candidate control signal. Then instead of implementing each of the controllers in the family as a separate dynamic system, one can often achieve the same results using a single controller with adjustable parameters, viz. the multicontroller.

This implementation strategy motivates the multirealization problem [4] [18]. In standard linear system realization, we only need to find a state space realization to realize one transfer function. For a multi-realization, we need to find a parameter-dependent state space description for a finite collection of systems, which may be those of the family of controllers.

Most literature on system realization deals with the implementation of a single linear time-invariant (LTI) system [3] [5] [8] [11] [20] [21] based on one of a state space description approach or matrix fraction description approach. Morse [12] presented some results for the multi-realization of several linear SISO systems in the context of examining MMAC for scalar plants. Papers [4] [18] investigate the multi-realization of several linear multiple input multiple output (MIMO) systems. The results are applicable to MMAC problems for linear MIMO plants. In this paper, we give a modest extension to the nonlinear case. Specifically, we

Dr. Steven W. Su and Prof. Hung T. Nguyen are with the Faculty of Engineering and Information Technology, University of Technology, Sydney, Australia. \{Steven.Su\}\{Hung. Nguyen\}@uts . edu.au

Prof. Brian D.O. Anderson is with Research School of Information Sciences and Engineering, The Australian National University, Australia and National ICT Australia, PO Box 8001, Canberra, ACT 2601, Australia. Brian. Andersoneanu.edu.au

Prof. Weidong Chen is with the Institute of Automation, Shanghai Jiao Tong University, China. wdchenes jtu.edu. cn consider the multi-realization of a finite set of feedback linearizable systems [7] [15].

In the next section, we recall the result on multi-realization of a set of linear SISO systems by state sharing and feedback. In Section III, we introduce the problem of feedback multirealization of a set of nonlinear systems, and present the result on minimal multi-realization of a set of feedback linearizable nonlinear systems. Two illustrative examples are given in Section IV.

\section{Multi-Realization FOR Linear SISO Systems}

Suppose that it is desired to implement a finite number of SISO linear proper rational systems with transfer functions $\kappa_{i}(s)=\frac{n_{i}(s)}{d_{i}(s)}(i \in \mathscr{I})$, where $\left(n_{i}(s), d_{i}(s)\right)$ are coprime polynomials. Assuming an upper bound $n$ for the McMillan Degree of the $\kappa_{i}(s)$, it is shown in [12] that we can always find an n-dimensional controllable pair $\left(A_{0}, b_{0}\right)$ with $\operatorname{Re}\left\{\lambda_{i}\left(A_{0}\right)\right\}<0$ such that $\left\{A_{0}+b_{0} k_{q_{i}}, b_{0}, c_{q_{i}}, d_{q_{i}}\right\}$ is a state space realization of each transfer function $\kappa_{i}(s)$, with corresponding adjustable parameters $k_{q_{i}} \in \mathscr{R}^{n}, c_{q_{i}} \in \mathscr{R}^{1 \times n}, d_{q_{i}} \in \mathscr{R}, i \in\{1,2, \ldots, N\}$. There is a MIMO generalization of the problem, see [4] [18] and we will return to this later.

\section{EXTENSION TO NONLINEAR SYSTEMS}

Firstly, we introduce some basic notation and facts of differential geometry drawn from [7] and [15].

\section{A. Some notations in differential geometry}

A smooth vector field $f$, defined on an open set $U$ of $\mathscr{R}^{n}$, can be intuitively interpreted as a smooth mapping assigning the $n$-dimensional vector $f(x)$ to each point $x$ of $U$. Suppose now that $d$ smooth vector fields $f_{1}, \cdots, f_{d}$ are given, all defined on the same open set $U$ and note that, at any fixed point $x$ in $U$, the vectors $f_{1}(x), \cdots, f_{d}(x)$ span a vector space (a subspace of the vector space in which all the $f_{i}(x)^{\prime} s$ are defined, i.e. a subspace of $\left.\mathscr{R}^{n}\right)$. Let this vector space, which depends on $x$, be denoted by $\Delta(x)$, i.e. set

$$
\Delta(x)=\operatorname{span}\left\{f_{1}(x), \cdots, f_{d}(x)\right\}
$$

and note that, in doing this, we have essentially assigned a vector space to each point $x$ of the set $U$. The object thus characterized, namely the assignment of the subspace spanned by the values at $x$ of some smooth vector fields defined on $U$, is called a smooth distribution.

Next, we recall some facts about Lie derivatives of functions (155-156 in [15]).

Given a smooth vector-field $X$ on $\mathscr{R}^{n}$ and a smooth function $h$ on $\mathscr{R}^{n}$ the Lie derivative of $h$ with respect to 
$X$ is the function

$$
L_{X} h(x)=X(h)(x)=\frac{\partial h}{\partial x}(x) \cdot X(x) .
$$

Similarly the functions $L_{X}^{k} h$ are defined as follows. By convention we set $L_{X}^{0} h(x)=h(x)$ and inductively for $k \geq 1$,

$$
L_{X}^{k} h(x)=L_{X}\left(L_{X}^{k-1} h\right)(x) .
$$

Analogously for a smooth mapping $h: \mathscr{R}^{n} \mapsto \mathscr{R}^{p}$ we define $L_{X}^{k} h(x)=L_{X}^{k} h$ component-wise, i.e. $L_{X}^{k} h=$ $\left(L_{X}^{k} h_{1}, \cdots, L_{X}^{k} h_{p}\right)^{T}$. For the nonlinear system

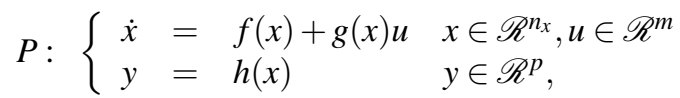

we introduce (in the local coordinates $x$ ) the mapping

$$
W^{k}(x)=\left[\begin{array}{l}
h(x) \\
L_{f} h(x) \\
\vdots \\
L_{f}^{k-1} h(x)
\end{array}\right]
$$

For any two vector-fields of $f$ and $g$ on $\mathscr{R}^{n}$, the Lie bracket $[f, g]$ is a vector field as given in [17]

$$
[f, g]=\frac{\partial g}{\partial x} f-\frac{\partial f}{\partial x} g
$$

We define the repeated Lie bracket $a d_{f}^{k} g, k=0,1,2, \cdots$, inductively as $a d_{f}^{k} g=\left[f, a d_{f}^{k-1} g\right], k \geq 1$, with $a d_{f}^{0} g=g$

A set of vector fields $\left\{X_{1}, \cdots, X_{m}\right\}$ is said to be involutive if there are scalar fields $\alpha_{i j k}$ such that

$$
\left[X_{i}, X_{j}\right]=\sum_{k=1}^{m} \alpha_{i j k} X_{k}
$$

A multivariable nonlinear system of the form (1) has a (vector) relative degree [7] $\left\{r_{1}, r_{2}, \cdots, r_{m}\right\}$ at a point $x_{0}$ if

i)

$$
L_{g_{j}} L_{f}^{k} h_{i}(x)=0
$$

for all $1 \leq j \leq m$, for all $1 \leq i \leq m$ for all $0 \leq k<r_{i}-1$, and for all $x$ in a neighbourhood of $x_{0}$, and

ii) the $m \times m$ matrix

$$
A(x)=\left[\begin{array}{lll}
L_{g_{1}} L_{f}^{r_{1}-1} h_{1}(x) & \cdots & L_{g_{m}} L_{f}^{r_{1}-1} h_{1}(x) \\
L_{g_{1}} L_{f}^{r_{2}-1} h_{2}(x) & \cdots & L_{g_{m}} L_{f}^{r_{2}-1} h_{2}(x) \\
\cdots & \cdots & \cdots \\
L_{g_{1}} L_{f}^{r_{m}-1} h_{m}(x) & \cdots & L_{g_{m}} L_{f}^{r_{m}-1} h_{m}(x)
\end{array}\right]
$$

is non-singular at $x=x_{0}$.

More detailed statements about differential geometry and fundamentals of nonlinear control can be found in [7], [9], and [15].

\section{B. Multi-realization problem for nonlinear systems}

We first define the problem of finding a multi-realization of a set of nonlinear systems as an extension of the linear multi-realization problem.

Definition 1: Assume that there are given a number of $m$-input $p$-output nonlinear systems $P_{i}(i \in\{1,2, \ldots, N\})$ described by

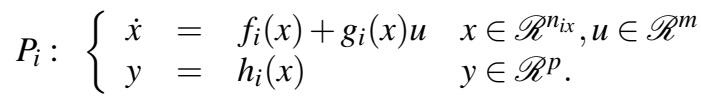

Provided that a state space description

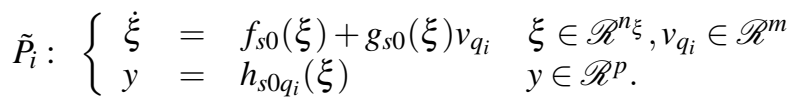

with coordinate transformation (a smooth invertible transformation $x=\Phi_{i}(\xi)$ in a neighborhood of $\xi_{0}$ [7]) and state feedback $\left(v_{q i}=\alpha_{q_{i}}(\xi)+\beta_{q_{i}}(\xi) u\right)$ can "realize" (locally or globally) each system $P_{i}$ (thus the functions $\alpha_{q_{i}}(\xi), \beta_{q_{i}}(\xi)$ and $h_{s o q_{i}}(\xi)$ are adjustable while $f_{s 0}$ and $g_{s 0}$ remain fixed), then we call the state space description (4) a multi-realization of the set of systems $P_{i}(i \in\{1,2, \ldots, N\})$. If the unforced system $\dot{\xi}=f_{s 0}(\xi)$ is asymptotically stable, we say that the state space description (4) is a stably-based multi-realization of the set of systems $P_{i}(i \in\{1,2, \ldots, N\})$.

Furthermore, if the dimension of the system $\tilde{P}_{i}\left(n_{\xi}\right)$ is the smallest of all such stably-based multi-realizations, then we call the state space description (4) a minimal stablybased multi-realization of the set of nonlinear systems $P_{i}$ $(i \in\{1,2, \ldots, N\})$.

Note 1: In the statement of Definition 1, the word "realize" means that system $\tilde{P}_{i}$ can be transformed into $P_{i}$ by means of feedback and change of coordinates in the state space.

The problem of multi-realization of nonlinear systems is obviously more complicated and difficult than in the linear case, especially the nonlinear minimal stably-based multi-realization problem. In the next section, we will only consider a special nonlinear multi-realization problem: the multi-realization of state equations of feedback linearizable nonlinear systems.

\section{The multi-realization of state equations of feedback lin- earizable nonlinear systems}

Problem 1: Assume that there are given a number of state equations (without output equations) of nonlinear systems $P_{i}$ $(i \in\{1,2, \ldots, N\})$ described by

$$
P_{i}: \quad \dot{x}=f_{i}(x)+g_{i}(x) u \quad x \in \mathscr{R}^{n_{i x}}, u \in \mathscr{R}^{m} .
$$

Find a state equation (6)

$$
\tilde{P}_{i}: \quad \dot{\xi}=A_{0} \xi+B_{0} v_{i} \quad \xi \in \mathscr{R}^{n_{\xi}}, v_{i} \in \mathscr{R}^{m} .
$$

with coordinate transformation (a smooth invertible transformation $x=\Phi_{i}(\xi)$ in a neighborhood of $\left.\xi_{0}\right)$ and state feedback $\left(v_{i}=\alpha_{i}(\xi)+\beta_{i}(\xi) u\right)$ to "realize" (locally or globally) each state equation (5), where the functions $\alpha_{i}(\xi)$ and $\beta_{i}(\xi)$ are adjustable. Further, $A_{0}$ is required to be stable (i.e. all 
eigenvalues should lie in the open left half plane) and be of smallest possible dimension.

Note 2: 1) The state feedback

$$
v_{i}=\alpha_{i}(\xi)+\beta_{i}(\xi) u
$$

in which

$\alpha_{i}(\xi)=\left[\begin{array}{c}\alpha_{i 1}(\xi) \\ \alpha_{i 2}(\xi) \\ \vdots \\ \alpha_{i m}(\xi)\end{array}\right], \quad \beta_{i}(\xi)=\left[\begin{array}{ccc}\beta_{i_{11}}(\xi) & \cdots & \beta_{i_{1 m}}(\xi) \\ \cdots & \cdots & \cdots \\ \beta_{i_{m 1}}(\xi) & \cdots & \beta_{i_{m m}}(\xi)\end{array}\right]$

are an $m \times 1$ vector and, respectively, an $m \times m$ matrix, and the entries of $\alpha_{i}(\xi)$ and $\beta_{i}(\xi)$ are smooth functions defined on an open subset of $\mathscr{R}^{n}$. In the following discussions, we also assume that the matrix $\beta_{i}(\xi)$ is nonsingular for all $\xi$. Accordingly, the feedback is called a "regular static state feedback" [7].

2) In the statement of Problem 1, the word "realize" means that the state equation (5) can be constructed by selecting some or all transformed (feedback and change of coordinates) states of the state equation (6). Hence, it is obvious that $n_{\xi} \geq n_{i x}, \forall i \in\{1,2, \cdots, N\}$.

In order to solve Problem 1, we present some results about feedback linearizable systems from [7].

Firstly, we introduce the so-called "State Space Exact Linearization Problem" [7].

Problem 2: Given a state equation (without output equation) of a nonlinear system $P$ described by

$$
P: \dot{x}=f(x)+g(x) u,
$$

and an initial state $x_{0}$, find (if possible), a neighborhood $U$ of $x_{0}$, a pair of feedback functions $\alpha(x)$ and $\beta(x)$ defined on $U$, a coordinate transformation $\xi=\Phi(x)$ also defined on $U$, a matrix $A \in \mathscr{R}^{n \times n}$ and a matrix $B \in \mathscr{R}^{n \times m}$, such that

$$
\begin{gathered}
{\left[\frac{\partial \Phi}{\partial x}(f(x)+g(x) \alpha(x))\right]_{x=\Phi^{-1}(\xi)}=A \xi} \\
{\left[\frac{\partial \Phi}{\partial x}(g(x) \beta(x))\right]_{x=\Phi^{-1}(\xi)}=B}
\end{gathered}
$$

and

$$
\operatorname{rank}\left(B A B \cdots A^{n-1} B\right)=n .
$$

The following results for the "State Space Exact Linearization Problem" are directly from [7].

Lemma 1: Suppose the matrix $g\left(x_{0}\right)$ has rank $m$. Then, the "State Space Exact Linearization Problem" is solvable if and only if there exist a neighborhood $U$ of $x_{0}$ and $m$ realvalued functions $h_{1}(x), \cdots, h_{m}(x)$ defined on $U$, such that the system

$$
\left\{\begin{aligned}
\dot{x} & =f(x)+g(x) u \\
y & =h(x)
\end{aligned}\right.
$$

has some relative degree $\left\{r_{1}, r_{2}, \cdots, r_{m}\right\}$ at $x_{0}$ and $r_{1}+r_{2}+$ $\cdots+r_{m}=n$.

Furthermore, the matrices $A$ and $B$ in equation (8) and equation (9) of Problem 2 could be in the form (Brunowsky canonical form):

$$
\begin{aligned}
& A=\operatorname{diag}\left\{A_{1}, A_{2}, \cdots, A_{m}\right\} \\
& B=\operatorname{diag}\left\{b_{1}, b_{2}, \cdots, b_{m}\right\}
\end{aligned}
$$

where $A_{i}$ is the $r_{i} \times r_{i}$ matrix

$$
A_{i}=\left[\begin{array}{ccccc}
0 & 1 & 0 & \ldots & 0 \\
0 & 0 & 1 & \ldots & 0 \\
\ldots & \ldots & \ldots & \ldots & \ldots \\
0 & 0 & 0 & \ldots & 1 \\
0 & 0 & 0 & \ldots & 0
\end{array}\right]
$$

and $b_{i}$ is the $r_{i} \times 1$ vector

$$
b_{i}=[0, \cdots, 0,1]^{T} \text {. }
$$

Proof: See Page 246-248 in [7].

Note 3: 1) From Lemma 1, we can see that the controllable pair $\{A, B\}$ of the feedback linearized system has controllability indices $\left\{r_{1}, r_{2}, \cdots, r_{m}\right\}$; this set is invariant under state feedback, input transformation, and coordinate transformation.

2) It is easily checked that the order of two transformations (state feedback and coordinate transformation) used to obtain the linear form can be interchanged (see Remark 2.1 in Page 158-159 of [7]).

The geometric conditions for the solution of "State Space Exact Linearization Problem" are presented in the following lemma [7].

Lemma 2: Suppose the matrix $g\left(x_{0}\right)$ has rank $m$. Then, there exists a neighborhood $U$ of $x_{0}$ and $m$ real-valued functions $\lambda_{1}(x), \lambda_{2}(x), \cdots, \lambda_{m}(x)$ defined on $U$, such that the system

$$
\left\{\begin{array}{l}
\dot{x}=f(x)+g(x) u \\
y=\lambda(x)
\end{array}\right.
$$

has some relative degree $\left\{r_{1}, r_{2}, \cdots, r_{m}\right\}$ at $x_{0}$, with

$$
r_{1}+r_{2}+\cdots+r_{m}=n
$$

if and only if

i) for each $0 \leq l \leq n-1$, the distribution $G_{l}$ defined below has constant dimension near $x_{0}$;

ii) the distribution $G_{n-1}$ has dimension $n$;

iii) for each $0 \leq l<n-1$, the distribution $G_{l}$ is involutive. Here,

$$
\begin{aligned}
& G_{0}=\operatorname{span}\left\{g_{1}, \cdots, g_{m}\right\} \\
& G_{1}=\operatorname{span}\left\{g_{1}, \cdots, g_{m}, a d_{f} g_{1}, \ldots, a d_{f} g_{m}\right\} \\
& \quad \cdots \\
& G_{l} \quad=\operatorname{span}\left\{a d_{f}^{k} g_{j}: 0 \leq k \leq l, 1 \leq j \leq m\right\} .
\end{aligned}
$$

Proof: See Page 249-256 in [7].

Note 4: (1)This lemma presents geometric conditions for the solvability of the "State Space Exact Linearization Problem".

(2)The relative degrees $r_{1}, r_{2}, \cdots, r_{m}$ are directly identified in terms of the dimensions of the distributions $G_{0}, G_{1}, \cdots, G_{n-2}$ (see Remark 2.7 in Page 256 of [7]). Therefore, the relative degrees $r_{1}, r_{2}, \cdots, r_{m}$ are invariant under feedback and coordinate transformation. Furthermore, the relative degrees are equal to the controllability indices of the controllable pair $\left(A_{0}, B_{0}\right)$ for the linearized systems (see Note 3). 
Now that the conditions for "State Space Exact Linearization Problem" are clear, the next step returns to the problem of multi-realization of a set of linear systems by using state feedback, input transformation and coordinate transformation.

Now, we give a definition for the minimal stably-based multi-realization (with input transformation) for a set of linear systems.

Definition 2: Assume that there are given a number of $m$ input $p$-output strictly proper real rational transfer function matrices $P_{i}(i \in\{1,2, \ldots, N\})$. Provided that there exist statevariable realizations $\left\{A_{0}+B_{0} K_{q_{i}}, B_{0} G_{q i}, C_{q_{i}}\right\}$ (with the pair $\left(A_{0}, B_{0}\right)$ being controllable) that can realize all the systems $P_{i}$ with adjustable parameters $C_{q_{i}}, K_{q_{i}}$ and $G_{q i}$, then we call $\left\{A_{0}+B_{0} K_{q_{i}}, B_{0} G_{q i}, C_{q_{i}}\right\}$ a multi-realization (with input transformation) of the set of systems $P_{i}(i \in\{1,2, \ldots, N\})$. If all eigenvalues of $A_{0}$ are in the left half plane, we say that $\left\{A_{0}+B_{0} K_{q_{i}}, B_{0} G_{q i}, C_{q_{i}}\right\}$ is a stably-based multi-realization (with input transformation) of the set of systems $P_{i}(i \in$ $\{1,2, \ldots, N\})$. Furthermore, if the dimension of $A_{0}$ is the smallest of all such stably-based multi-realizations, then we call $\left\{A_{0}+B_{0} K_{q_{i}}, B_{0} G_{q i}, C_{q_{i}}\right\}$ a minimal stably-based multirealization (with input transformation) of the set of systems $P_{i}(i \in\{1,2, \ldots, N\})$. We now develop preliminary results used in proving the first Theorem below, which characterizes the minimal dimension $A_{0}$ in a stably-based multi-realization.

Lemma 3: Assume given a number of $m$-input $p$-output strictly proper linear systems $P_{i}(i \in\{1,2, \ldots, N\})$. Then there exists a controllable pair $\left(A_{0}, B_{0}\right)\left(A_{0} \in \mathscr{R}^{n \times n}, B_{0} \in \mathscr{R}^{n \times m}\right)$, and appropriately dimensioned real matrices $C_{q_{i}}, K_{q_{i}}$ and $G_{q_{i}}$ (for $i \in\{1,2, \ldots, N\}$ ) such that $A_{0}$ is stable, and $\left\{A_{0}+\right.$ $\left.B_{0} K_{q_{i}}, B_{0} G_{q i}, C_{q_{i}}\right\}$ is a controllable realization of system $P_{i}$ (for $i \in\{1,2, \ldots, N\}$ ), if and only if there exists a state space realization $\left\{A_{i} \in \mathscr{R}^{n \times n}, B_{i} \in \mathscr{R}^{n \times m}, C_{i} \in \mathscr{R}^{p \times n}\right\}$ (where the pair $\left(A_{i}, B_{i}\right)$ is controllable, and $B_{i}$ has full column rank.) for each system $P_{i}$ such that all controllable pairs $\left(A_{i}, B_{i}\right)$ (for each $i \in\{1,2, \ldots, N\}$ ) have identical controllability indices sets as $\left\{d_{1}, d_{2}, \ldots, d_{m}\right\}$ (without ordering requirement).

Proof: See Theorem 3 and its related remarks in Pages 260-261 of [16]. The unordered controllability indices are invariant under feedback, input transformation and coordinate transformation. This shows the condition of the theorem is necessary.

Now, we prove the sufficiency. According to item (1)(9) in Page 507-508 of [8] (and Theorem 3 in Page 260261 of [16]), we can find a controller form realization $\left\{A_{0}^{\prime}+B_{0} K_{q_{i}}^{\prime}, B_{0} G_{q i}, C_{q_{i}}\right\}$ for each system $P_{i}$ with $\left\{A_{0}^{\prime}, B_{0}\right\}$ controllable by adjusting parameters $K_{q_{i}}^{\prime}, G_{q i}$ and $C_{q_{i}}$. Furthermore, if $A_{0}^{\prime}$ is not stable, we can select a stable $A_{0}$ and a new adjustable feedback gain matrix $K_{q_{i}}$ such that $A_{0}^{\prime}+B_{0} K_{q_{i}}^{\prime}=A_{0}+B_{0} K_{q_{i}}$ because $\left\{A_{0}^{\prime}, B_{0}\right\}$ is controllable. Thus, we can construct a state-variable description $\left\{A_{0}+\right.$ $\left.B_{0} K_{q_{i}}, B_{0} G_{q i}, C_{q_{i}}\right\}$ which is a (multi-) realization of system $P_{i}$ (for $i \in\{1,2, \ldots, N\}$ ).

Lemma 3 provides the necessary and sufficient condition for multi-realization of a set of linear systems by a state-variable realization $\left\{A_{0}+B_{0} K_{q_{i}}, B_{0} G_{q i}, C_{q_{i}}\right\}$ with the dimension of $A_{0}$ fixed to $n$. If the dimension of $A_{0}$ is not fixed to $n$, the necessary and sufficient condition in Lemma 3 can be fulfilled by dimension augmentation of each controllable pair $\left(A_{i}, B_{i}\right)$ to ensure all $\left(A_{i}, B_{i}\right)$ (for each $i \in\{1,2, \ldots, N\}$ ) have identical controllability indices sets as $\left\{d_{1}, d_{2}, \ldots, d_{m}\right\}$ (without ordering requirement). Certainly, the minimal dimension augmentation is desired, and this is not difficult to achieve. The key to doing this is the following two lemmas; the first of these is only used within the proof of the second.

Lemma 4: Let $\left\{d_{i 1}, d_{i 2}\right\}$ for $i=1,2, \ldots, N$ denote $N$ pairs of positive integers. Let $\left\{r_{i 1}, r_{i 2}\right\}$ denote the pair $\left\{d_{i 1}, d_{i 2}\right\}$ possibly reordered, with $r_{i 1} \leq r_{i 2}$. Then,

$$
\sum_{j=1}^{2} \max _{1 \leq i \leq N}\left(d_{i j}\right) \geq \sum_{j=1}^{2} \max _{1 \leq i \leq N}\left(r_{i j}\right)
$$

Proof: Denote

$$
\begin{aligned}
& s_{d_{1}}=\max _{1 \leq i \leq N}\left(d_{i 1}\right), \\
& s_{d_{2}}=\max _{1 \leq i \leq N}\left(d_{i 2}\right) .
\end{aligned}
$$

Without loss of generality, assume $s_{d_{1}} \geq s_{d_{2}}$. Then it is obvious $s_{d_{1}}=\max _{1 \leq i \leq N}\left(r_{i 1}, r_{i 2}\right)=\max _{1 \leq i \leq N}\left(r_{i 2}\right)$, and $s_{d_{2}} \geq$ $\max _{1 \leq i \leq N}\left(r_{i 1}\right)$.

Hence, we have

$$
s_{d_{1}}+s_{d_{2}}=\sum_{j=1}^{2} \max _{1 \leq i \leq N}\left(d_{i j}\right) \geq \sum_{j=1}^{2} \max _{1 \leq i \leq N}\left(r_{i j}\right) .
$$

Lemma 5: Let $\mathscr{D}_{i}=\left\{d_{i 1}, d_{i 2}, \cdots, d_{i m}\right\}$ for $i=1,2, \ldots, N$ denote $N$ sets of $m$ positive integers, and let $\mathscr{R}_{i}=$ $\left\{r_{i 1}, r_{i 2}, \cdots, r_{i m}\right\}$ denote an ordering in which the $r_{i j}$ increase with $j$. Then,

$$
\sum_{j=1}^{m} \max _{1 \leq i \leq N}\left(r_{i j}\right) \leq \sum_{j=1}^{m} \max _{1 \leq i \leq N}\left(d_{i j}\right) .
$$

Proof: For each set $\mathscr{D}_{i}$ and a fixed integer $q \in$ $\{1,2, \cdots, m\}$, denote

$$
s_{d_{q}}=\max _{1 \leq i \leq N}\left(d_{i q}\right), \quad i \in\{1,2, \cdots, N\} .
$$

Then the right hand side of (16) can be rewritten as

$$
\sum_{j=1}^{m} \max _{1 \leq i \leq N}\left(d_{i j}\right)=\sum_{j=1}^{m} s_{d_{j}} .
$$

For any two fixed integers $k, l \in\{1,2, \cdots, m\}$, we have

$$
s_{d_{k}}+s_{d_{l}}=\max _{1 \leq i \leq N}\left(d_{i k}\right)+\max _{1 \leq i \leq N}\left(d_{i l}\right), \quad i \in\{1,2, \cdots, N\} .
$$

Without loss of generality, assume $s_{d_{k}} \geq s_{d_{l}}$. If for some $\mathscr{D}_{i}$, $d_{i k} \geq d_{i l}$, keep $d_{i k}$ in the $k_{t h}$ position and $d_{i l}$ in the $l_{t h}$ position. Otherwise, if $d_{i k} \leq d_{i l}$, adjust $d_{i k}$ to in the $l_{t h}$ position and $d_{i l}$ to in the $k_{t h}$ position. We denote the reordered set as $\tilde{\mathscr{D}}_{i}=\left\{\tilde{d}_{i 1}, \tilde{d}_{i 2}, \cdots, \tilde{d}_{i m}\right\}$ and set

$$
s_{\tilde{d}_{q}}=\max _{1 \leq i \leq N}\left(\tilde{d}_{i q}\right), i \in\{1,2, \cdots, N\} .
$$

According to Lemma 4, we have

$$
s_{\tilde{d}_{k}}+s_{\tilde{d}_{l}} \leq s_{d_{k}}+s_{d_{l}} .
$$


Repeat this adjustment for every two integers $k, l \in$ $\{1,2, \cdots, m\}$. Then, after all these adjustments, the set $\mathscr{D}_{i}$ will have been re-ordered as set $\mathscr{R}_{i}$. Hence, we have

$$
\sum_{j=1}^{m} \max _{1 \leq i \leq N}\left(r_{i j}\right) \leq \sum_{j=1}^{m} \max _{1 \leq i \leq N}\left(d_{i j}\right) .
$$

A more general case of the above lemma appears as Theorem D.7.a in Page 155 of [10].

Theorem 1: Assume given $N$ distinct $m$-input $p$-output systems $P_{i}(i \in\{1,2, \cdots, N\})$ described by $A_{i} \in \mathscr{R}^{n_{i} \times n_{i}}, B_{i} \in$ $\mathscr{R}^{n_{i} \times m}$ and $C_{i} \in \mathscr{R}^{p \times n_{i}}$, and suppose that the pairs $\left(A_{i}, B_{i}\right)$ and $\left(C_{i}, A_{i}\right)$ are controllable and observable, and $B_{i}$ has full column rank. Further, assume the controllability indices for the pair $\left(A_{i}, B_{i}\right)$ are $d_{i 1}, d_{i 2}, \cdots, d_{i m}$, and define the ordered set $\left\{r_{i 1}, r_{i 2}, r_{i 3}, \ldots\right\}$ to be the set of controllability indices reordered so that

$$
r_{i 1} \leq r_{i 2} \leq \cdots \leq r_{i m}, \forall i \in\{1,2, \cdots, N\}
$$

Then the dimension of the minimal stably-based multirealization (with input transformation) of the set of systems $P_{i}(i \in\{1,2, \cdots, N\})$ is equal to

$$
\bar{n}=\sum_{j=1}^{m} \max _{1 \leq i \leq N}\left(r_{i j}\right) .
$$

Proof: The proof of this theorem is based on Lemma 3 and Lemma 5. Based on Lemma 3 we can prove the existence of a stably-based multirealization with dimension $\bar{n}$. From Lemma 5 we can derive that $\bar{n}$ is minimal. Specifically, according to Lemma 3 , to obtain a stably-based multirealization with the fixed dimension $\bar{n}$, we need to find a state space realization, $\left\{\bar{A}_{i} \in \mathscr{R}^{\bar{n} \times \bar{n}}, \bar{B}_{i} \in \mathscr{R}^{\bar{n} \times m}, \bar{C}_{i} \in \mathscr{R}^{p \times \bar{n}}\right\}$ (with the pair $\left(\bar{A}_{i}, \bar{B}_{i}\right)$ controllable, and $\bar{B}_{i}$ of full column rank) for each system $P_{i}$ such that all the controllable pairs $\left(\bar{A}_{i}, \bar{B}_{i}\right) \quad(i \in\{1,2, \ldots, N\})$ have an identical controllability indices set as $\left\{\bar{d}_{1}, \bar{d}_{2}, \ldots, \bar{d}_{m}\right\}$ (without order requirement) and $\bar{d}_{1}+\bar{d}_{2}+\cdots+\bar{d}_{m}=\bar{n}$. Now, if we define $\bar{d}_{j}=\max _{1<i<N}\left(r_{i j}\right)$, then it is easy to check that $\bar{d}_{1}+\bar{d}_{2}+\cdots+\bar{d}_{m}=\bar{n}$. For any system $P_{i}$, the ordered set $\left\{r_{i 1}, r_{i 2}, r_{i 3}, \ldots r_{i m}\right\}$ of controllability indices for the pair $\left(A_{i}, B_{i}\right)$ satisfies $r_{i j} \leq \bar{d}_{j}$. Then it is possible to increase controllability indices by dimension augmentation so that the pair $\left(A_{i}, B_{i}\right)$ is replaced by a pari $\left(\bar{A}_{i}, \bar{B}_{i}\right)$ for which the controllability indices are $\left\{\bar{d}_{1}, \bar{d}_{2}, \cdots, \bar{d}_{m}\right\}$. To see this, consider a right polynomial matrix fraction description (MFD) $N_{E i}(s) D_{E i}^{-1}(s)$ of system $P_{i}$, where $D_{E i}(s)$ is a Popov polynomial matrix [8]. Based on Theorem 1 in [4], the augmentation of controllability indices can be implemented by right multiplication using a stable diagonal polynomial matrix of both $N_{E i}(s)$ and $D_{E i}(s)$. The reordering of the controllability indices can be achieved by simply using an input transformation matrix $G_{q i}$. By using $G_{q i}$, the pivot indices of the Popov polynomial matrix $D_{E i}(s)$ can be adjusted so that the controllability indices are reordered (In Example 1 of this paper, see the next section, the controllability indices of the linearized model of the first system $P_{1}$ are increased by dimension augmentation. The controllability indices of the linearized model of the second system $P_{2}$ are reordered by using an input transformation).

Now that the existence of an $\bar{n}$-th order stably-based multirealization (with input transformation) has been proved, we only need to prove $\bar{n}$ is minimal. The minimality is however easily proved by using Lemma 5, which deals with an equivalent problem of finding the minimal summation of several sets of integer numbers. Specifically, if we treat each $d_{i j}$ in Lemma 5 as the controllability indices of system $P_{i}$ in this theorem, then the proof of minimality is straightforward.

Lemma 1 and 2 solve the "State Space Exact Linearization Problem". They provide the conditions based on which a nonlinear system can be linearized by using "regular static state feedback" and coordinate transformation. Then, based on Lemma 3 and Theorem 1, we can give the answer for Problem 1, requiring multi-realization of state equations of a finite set of feedback linearizable nonlinear systems.

Theorem 2: (Main Result) Suppose the matrices $g_{i}\left(x_{0}\right), \forall i \in\{1,2, \cdots, N\}$ in equation (5) of Problem 1 have rank $m$. Then, Problem 1 is solvable if and only if the following equivalent conditions (a) or (b) hold:

(a) For each system $P_{i}$ (described by equation (5)), there exist a neighborhood $U$ of $x_{0}$ and $m$ real-valued functions $h_{i 1}(x), \cdots, h_{i m}(x)$ defined on $U$, such that the system

$$
\left\{\begin{aligned}
\dot{x} & =f_{i}(x)+g_{i}(x) u \\
y & =h_{i}(x)
\end{aligned}\right.
$$

has some relative degree $\left\{r_{i 1}, r_{i 2}, \cdots, r_{i m}\right\}$ at $x_{0}$ and $r_{i 1}+r_{i 2}+$ $\cdots,+r_{i m}=n_{i}$.

(b) i) for each $i \in\{1,2, \cdots, N\}$ and $l \in\{1,2, \cdots, n-1\}$, the distribution $G_{i l}$ defined below has constant dimension near $x_{0}$;

ii) the distribution $G_{i, n-2}(i \in\{1,2, \cdots, N\})$ has dimension $n$;

iii) for each $i \in\{1,2, \cdots, N\}$ and $l \in\{1,2, \cdots, n-2\}$, the distribution $G_{i l}$ is involutive.

Here,

$$
\begin{aligned}
G_{i 0} & =\operatorname{span}\left\{g_{i 1}, \cdots, g_{i m}\right\} \\
G_{i 1} & =\operatorname{span}\left\{g_{i 1}, \cdots, g_{i m}, a d_{f_{i}} g_{i 1}, \ldots, a d_{f_{i}} g_{i m}\right\} \\
& \ldots \\
G_{i l} & =\operatorname{span}\left\{a d_{f_{i}}^{k} g_{i j}: 0 \leq k \leq l, 1 \leq j \leq m\right\} .
\end{aligned}
$$

Furthermore, the smallest possible dimension for equation (6) to realize (locally or globally) each state equation (5) is equal to

$$
\bar{n}=\sum_{i=1}^{N} \max _{1 \leq j \leq m}\left(r_{i j}\right) .
$$

Proof: From Lemma 1 and 2, we conclude that the set of nonlinear state space equations (see equation (5) ) can be expressed as linear state space equations (with feedback and coordinate transformation) if and only if Conditions i), ii) and iii) are satisfied. Then, according to Lemma 3 and Theorem 1, we can find a minimal stably-based multi-realization for these linearized systems. Further, considering that the state feedback and coordinate transformation are interchangeable 
(see Note 3), we conclude that Problem 1 is solvable if the conditions of (a) and (b) hold.

Because Conditions i), ii) and iii) of b) are necessary for the set of nonlinear state space equations (see equation (5) ) to be linearizable using feedback and coordinate transformation, they are necessary for the solvability of Problem 1

\section{EXAMPLE}

We build a block diagram (Fig. 1) to illustrate the implementation of the proposed multirealization structure. Here, we use the symbols and notations given in Definition 1. In distinction to typical labelling conventions in a linear block diagram, in Fig. 1, a block " $f(\cdot)$ " means " $y=f(u)$ ", while a block " $f(\xi)$ " means " $y=f(\xi) \cdot u$ ". This structure has been implemented by using Matlab Simulink for the control in the following example.

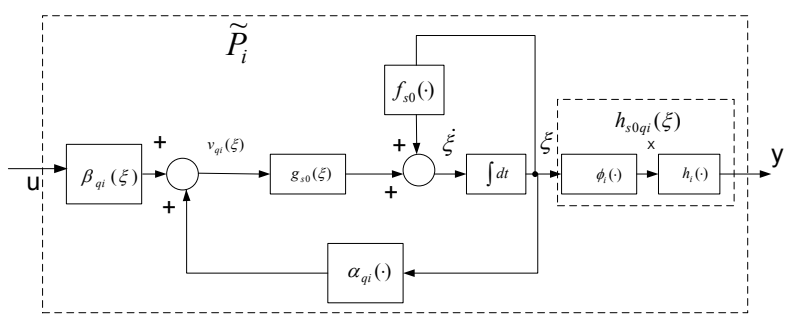

Fig. 1. The block diagram of the proposed stably-based state sharing multirealization structure.

Example 1: We first consider the multirealization of nonlinear lag-lead networks proposed in [19]. As pointed in [19], sometimes multiple desired system specifications cannot be satisfied simultaneously using classical linear control (purely linear compensation) methods. For example, in order to limit the absolute amount of overshoot in a step response, the classical linear system is often designed to be overdamped (with slow transient response), i.e. with linear control simultaneous constraints on step response rise time and overshoot may not be achievable. In this example, we will show it is possible to decrease both rise time and overshoot by switching between different types of controllers. The main strategy involves using a fast controller during the rise period, and switching to a slow controller when output is close to steady state. This paper does not attempt to prove that the control performance of switching between nonlinear controllers is superior to that of linear controllers. However, it does provide more options for switching controller selection and proposed stably-based multirealization approach to implement switching between controllers which are not necessarily linear.

Example 1 in [19] provided three controllers: $C_{1}$ (linear compensator), $C_{2}$ (nonlinear lag compensator) and $C_{3}$ (nonlinear lag-lead compensator). That is:

$$
C_{1}:\left\{\begin{array}{l}
\dot{x}=-\frac{1}{\alpha} x+\frac{k}{\alpha} u \\
y=x
\end{array}\right.
$$

$$
\begin{gathered}
C_{2}:\left\{\begin{array}{l}
\dot{x}=-\frac{1}{\alpha} x+\frac{k}{\alpha}\left(u-\frac{1}{k} x^{3}\right) \\
y=x,
\end{array}\right. \\
C_{3}:\left\{\begin{array}{l}
\dot{x}=-\frac{1}{\alpha} x+\frac{k}{\alpha}\left(u-\frac{1}{k} x^{3}\right) \\
y=(1-\beta)\left[x+x^{3}\right]-k \beta u .
\end{array}\right.
\end{gathered}
$$

In this particular example, the slow controller is a nonlinear lag compensator $C_{2}$ (equation (2.1) in [19]). The other two controllers $C_{1}$ and $C_{3}$ are relatively fast. We implement two switching controllers. The first one is the controller which switches from the linear compensator $C_{1}$ (fast controller) to the nonlinear lag compensator $C_{2}$. The second one switches from the nonlinear lag-lead compensator $C_{3}$ (a fast controller given in equation (2.2) in [19]) to the nonlinear lag compensator $C_{2}$. The switching between controllers happens when the system output is close to the reference input.

It is easy to check the relative degrees of these three controllers are all less than 1 . Thus, the minimal dimension of the multirealization is $\bar{n}=1$.

Then $\tilde{P}_{i}$ (see Definition 1 ) can be easily constructed as follows:

$$
\dot{\xi}=-\frac{1}{\alpha} \xi+\frac{k}{\alpha} v_{q i}
$$

To implement $C_{1}$, we can use the following settings:

$$
\left\{\begin{array}{l}
v_{q 1}=\alpha_{q 1}(\xi)+\beta_{q 1}(\xi) u=u \\
x=\phi_{1}(\zeta)=\zeta \\
y=h_{1}(x)=x=h_{s o q 1}(\zeta)=\zeta
\end{array}\right.
$$

To implement $C_{2}$, we can use the following settings:

$$
\left\{\begin{array}{l}
v_{q 2}=\alpha_{q 2}(\xi)+\beta_{q 2}(\xi) u=-\frac{1}{k} \zeta^{3}+u \\
x=\phi_{2}(\zeta)=\zeta \\
y=h_{2}(x)=x=h_{\text {soq2 }}(\zeta)=\zeta
\end{array}\right.
$$

To implement $C_{3}$, we can use the following settings:

$$
\left\{\begin{array}{c}
v_{q 3}=\alpha_{q 3}(\xi)+\beta_{q 3}(\xi) u=-\frac{1}{k} \zeta^{3}+u \\
x=\phi_{3}(\zeta)=\zeta \\
y=h_{3}(x)=(1-\beta)\left[x+x^{3}\right]-k \beta u \\
=h_{\text {soq } 3}(\zeta)=(1-\beta)\left[\zeta+\zeta^{3}\right]-k \beta u .
\end{array}\right.
$$

It should be noted that as the output equation $h_{i}(\cdot)$ does not change $(y=x)$ for the first switching controller, bumpless transfer can be achieved. For the second switching controller, on the other hand, as the output equation changes during switching, bumpless transfer cannot be guaranteed. However, the state values are continuous during switching for the two switching controllers as their states are shared. It should be noted that a (control affine) single state controller always satisfies the conditions in Theorem 2. In practice, low order controllers are often the first options.

During simulation, the reference input is selected as 5, and the switching point is selected at 4.5 ( 90 percent of reference input). The parameters of the three controllers and system models are all the same as in Example 1 of [19], which ensures the stability of the closed loop system under one of these three controllers. However, there are more options for nonlinear compensator design. For example, the nonlinear function $f(\sigma)$ defined in [19] is not restricted to being a 

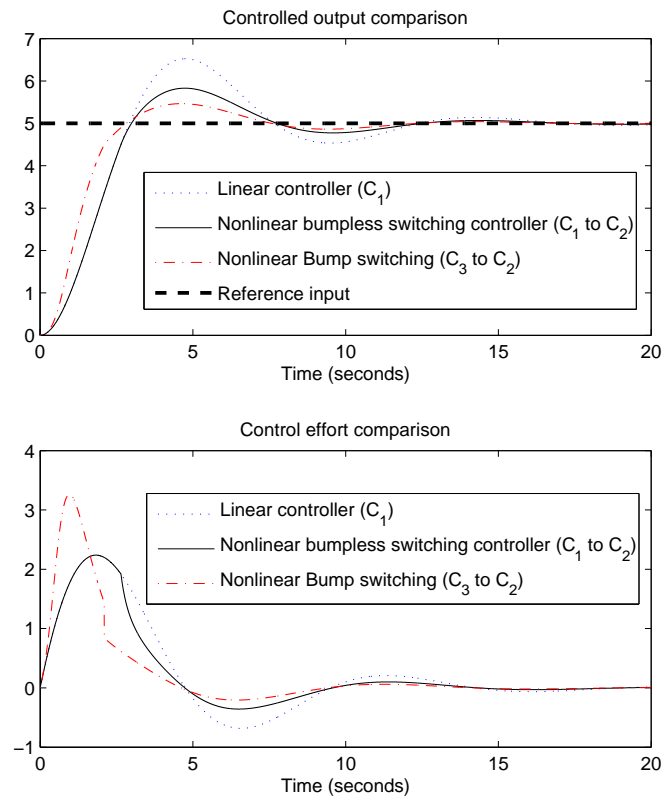

Fig. 2. Control performance comparison.

cubic function. It can be any static passive function, which gives more freedom for the design of multiple model adaptive control and switching control.

Control performance comparison are shown in Fig. 2. It can be seen that the performance of the two switching controllers outweighs the classical linear controller. The second switching controller achieves the best control performance. However, bump transfer appears for this switching controller and control effort is bigger, which may be an undesired property for some control system design. As the proposed stably-based state sharing multirealization approach can efficiently implement the switching between not necessarily linear controllers, so evidently this paper at least provides more choice for switching control or multiple model adaptive controller design.

The first example is for SISO case. In engineering practice, most current nonlinear controllers are still SISO. For the nonlinear MIMO case, we use the following artificial example to further illustrate the proposed implementation approach.

Example 2: We show the multirealization of the following two multivariable systems (the symbols and notations are defined as in Definition 1):

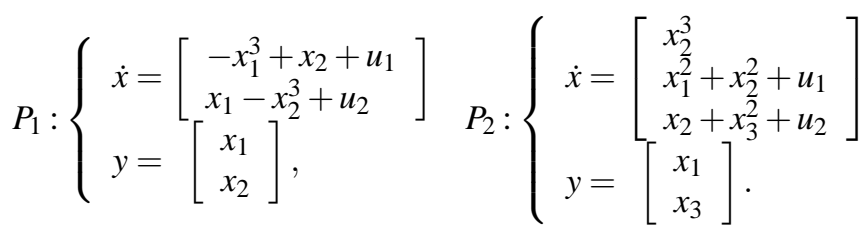

For system $P_{1}$, the relative degrees associated with output channels $\left(r_{1}\right.$ and $\left.r_{2}\right)$ satisfy the condition $r_{1}+r_{2}=1+1=n_{1}$. For system $P_{2}$, the relative degrees $\left(r_{1}\right.$ and $\left.r_{2}\right)$ satisfy the condition $r_{1}+r_{2}=2+1=n_{2}$ as well. Thus, both $P_{1}$ and $P_{2}$ are linearizable. According to Theorem 2, the minimal order of the multi-realization is $\bar{n}=3$.

Therefore, we construct a third order stable system $\tilde{P}_{i}$ (see Definition 1) with its controllability indices as $d_{1}=1$ and $d_{2}=2$ (the controllability indices are increasingly ordered):

$$
\begin{aligned}
\dot{\xi} & =f_{s 0}(\xi)+g_{s 0}(\xi) v_{q i} \\
& =\left[\begin{array}{ccc}
-3 & 0 & 0 \\
0 & 0 & 1 \\
0 & -1 & -2
\end{array}\right]\left[\begin{array}{l}
\xi_{1} \\
\xi_{2} \\
\xi_{3}
\end{array}\right]+\left[\begin{array}{ll}
1 & 0 \\
0 & 0 \\
0 & 1
\end{array}\right]\left[\begin{array}{l}
v_{q i 1} \\
v_{q i 2}
\end{array}\right] .
\end{aligned}
$$

By using state transformation and static feedback as shown in [7], we can linearize the two systems and obtain the following settings to implement the two systems.

To implement $P_{1}$, we can use the following settings:

$$
\left\{\begin{array}{l}
v_{q 1}=\alpha_{q 1}(\xi)+\beta_{q 1}(\xi) u \\
=\left[\begin{array}{c}
3 \xi_{1}-\xi_{1}^{3}+\xi_{2}+\xi_{3} \\
\xi_{1}+\xi_{2}+\xi_{3}-\left(\xi_{2}+\xi_{3}\right)^{3}
\end{array}\right]+\left[\begin{array}{ll}
1 & 0 \\
0 & 1
\end{array}\right]\left[\begin{array}{l}
u_{1} \\
u_{2}
\end{array}\right], \\
x=\phi_{1}(\xi)=\left[\begin{array}{lll}
1 & 0 & 0 \\
0 & 1 & 1 \\
0 & 0 & 1
\end{array}\right]\left[\begin{array}{l}
\xi_{1} \\
\xi_{2} \\
\xi_{3}
\end{array}\right], \\
y=h_{1}(x)=\left[\begin{array}{l}
x_{1} \\
x_{2}
\end{array}\right]=h_{\text {soq } 1}(\xi)=\left[\begin{array}{lll}
1 & 0 & 0 \\
0 & 1 & 1
\end{array}\right]\left[\begin{array}{l}
\xi_{1} \\
\xi_{2} \\
\xi_{3}
\end{array}\right] .
\end{array}\right.
$$

Then, the implemented system is

$$
\left\{\begin{array}{l}
\dot{x}=\left[\begin{array}{c}
-x_{1}^{3}+x_{2}+u_{1} \\
x_{1}-x_{2}^{3}+u_{2} \\
x_{1}-x_{3}-x_{3}^{3}+u_{2}
\end{array}\right] \\
y=\left[\begin{array}{c}
x_{1} \\
x_{2}
\end{array}\right]
\end{array}\right.
$$

From the above equation, we can see that state $x_{3}$ is unobservable but stable. The reason is the linearized model of system $P_{1}$ needs to be augmented so that its controllability indices match with those of $\tilde{P}_{i}$. At the same time, the stability of the augmented unobservable state is also guaranteed.

To implement $P_{2}$, we can use the following settings:

$$
\left\{\begin{array}{l}
v_{q 2}=\alpha_{q 2}(\xi)+\beta_{q 2}(\xi) u \\
=\left[\begin{array}{c}
\xi_{3}^{\frac{1}{3}}+\xi_{1}^{2}+3 \xi_{1} \\
3 \xi_{2}^{2} \xi_{3}^{\frac{2}{3}}+3 \xi_{3}^{\frac{4}{3}}+\xi_{2}+2 \xi_{3}
\end{array}\right]+\left[\begin{array}{cc}
0 & 1 \\
3 \xi_{3}^{\frac{2}{3}} & 0
\end{array}\right]\left[\begin{array}{l}
u_{1} \\
u_{2}
\end{array}\right], \\
x=\phi_{2}(\xi)=\left[\begin{array}{l}
\xi_{2} \\
\xi_{3}^{\frac{1}{3}} \\
\xi_{1}
\end{array}\right], \\
y=h_{2}(x)=\left[\begin{array}{l}
x_{1} \\
x_{3}
\end{array}\right]=h_{\text {soq } 2}(\xi)=\left[\begin{array}{lll}
0 & 1 & 0 \\
1 & 0 & 0
\end{array}\right]\left[\begin{array}{l}
\xi_{1} \\
\xi_{2} \\
\xi_{3}
\end{array}\right] .
\end{array}\right.
$$

In the above implementation, the input transformation $\beta_{q 2}(\xi)$ is designed to reorder the controllability indices of $P_{2}$ so that they match with those of $\tilde{P}_{i}$. However, it should be emphasized that in order to ensure the feedback is "regular static state feedback" [7] as assumed in Note 2, it is required that $\xi_{3} \neq 0$. 


\section{CONCLUSION}

In this paper, the problem of multi-realization of a set of linear SISO systems is reviewed. Then, the problem of multi-realization of a set of nonlinear systems is introduced. A minimal stably-based multi-realization of state equations of feedback linearizable nonlinear systems is achieved. We believe these results will make multiple model adaptive control and swithing control more efficient and practical.

\section{ACKNOWLEDGMENTS}

The first author would like to express his best thanks to Dr. Thomas Brinsmead (Division of Energy Technology, CSIRO, Australia) for the valuable discussions and suggestions. The work of Prof. Brian Anderson has been supported by ARC Discovery Project Grant DP0664427.

\section{REFERENCES}

[1] B. D. O. Anderson, T. Brinsmead, D. Liberzon, and A. S. Morse. Multiple model adaptive control with safe switching. Special issue of the International Journal of Robust and Nonlinear Control on Adaptive Control with Confidence, 8:446-470, 2001.

[2] B. D. O. Anderson, T. S. Brinsmead, F. de Bruyne, J. P. Hespanha, D. Liberzon, and A. S. Morse. Multiple model adaptive control. i. finite controller coverings. International Journal of Robust and Nonlinear Control, 10:909-929, 2000.

[3] B.D.O. Anderson and M.R. Gevers. On the minimality of feedback realisations. International Journal of Control, 37(1):145-158, 1983.

[4] B.D.O. Anderson, S.W. Su, and T.S. Brinsmead. Multi-realization of linear multi-variable systems. IEEE Transaction on Circuits and Systems II, 52(8):442-446, 2006.

[5] J. L. Casti. Dynamical systems and their applications: linear theory. Academic Press Inc, U.S.A., 1977.

[6] J.P. Hespanha, D. Liberzon, A.S. Morse, B.D.O. Anderson, T.S Brinsmead, and D.F. Bruyne. Multiple model adaptive control, part 2: Switching. International Journal of Robust and Nonlinear Control, 11:479-496, 2001.

[7] A. Isidori. Nonlinear Control Systems. Springer-Verlag, New York, 1989.

[8] T. Kailath. Linear systems. Prentice-Hall Inc, New Jersey, 1980.

[9] R. Marino and P. Tomei. Nonlinear Control Design: Geometric, Adaptive and Robust. Prentice Hall, London, 1995.

[10] A.W. Marshall and I. Olkin. Inequalities: Theory of majorization and its applications. Academic Press, New York, 1979.

[11] A. S. Morse. Structural invariants of linear multivariable systems. SIAM J. Control, 8:446-465, 1973.

[12] A.S. Morse. Control using logic-based swithing. In Alberto Isidori, editor, Trends in control: A European perspective, pages 69-113. Springer-Verlag, 1995.

[13] A.S. Morse. A bound for the disturbance-to-tracking-error gain of a supervised set-point control system. In D. Normand Cyrot, editor, Perspectives in Control, Theory and Applications, pages 2341. Springer-Verlag, 1998.

[14] K.S. Narendra and J. Balakrishnan. Adaptive control using multiple models. IEEE Transactions on Auto. Control, pages 42(2):171-187, February 1998.

[15] H. Nijmeijer and A.J.Van Der Schaft. Nonlinear Dynamical Control Systems. Springer Verlag, New York, 1989.

[16] V.M. Popov. Invariant description of linear, time-invariant controllable systems. SIAM J. Contr., 10:252-264, 1972 May.

[17] R. Su. On the linear equivalents of nonlinear systems. Syst. Contr. Lett., 2:48-52, 1982.

[18] S.W. Su, B.D.O. Anderson, and T.S. Brinsmead. The minimal multirealization of linear multi-variable systems. IEEE Transaction on Automatic Control, 51(4):690-695, 2006.

[19] D. Williamson. Nonlinear compensation of linear processes. Automatica, 15:601-608, 1979.

[20] W. A. Wolovich. Linear Multivariable System. Springer-Verlag, New York, 1974.
[21] Y. Yasuda. Feedback realisations in linear multivariable systems: model-following with stability and disturbance rejection. International Journal of Control, 42(5):1049-1070, 1985. 